\title{
Assessment of Microcirculatory Status Based on Stimulation of Myogenic Oscillations by Transient Ischemia: From Health to Disease
}

This article was published in the following Dove Press journal: Vascular Health and Risk Management

Jerzy Gebicki (1) ${ }^{1,2}$

Andrzej Marcinek (D) ${ }^{1,2}$

Jacek Zielinski ${ }^{3}$

'Institute of Applied Radiation Chemistry, Lodz University of Technology, Lodz, Poland; ${ }^{2}$ Angionica Ltd, Lodz, Poland; ${ }^{3}$ Department of Athletics, Strength and Conditioning, Poznan University of Physical Education, Poznan, Poland
Correspondence: Jerzy Gebicki Institute of Applied Radiation Chemistry, Lodz University of Technology, Lodz, 90924, Poland

$\mathrm{Tel}+4842$ 63| 3|7|

Email jerzy.gebicki@p.lodz.pl
The skin is the largest organ of the human body, characterized by exceptional metabolism. The epidermal layer of the skin is not directly vascularized, and oxygen and nutrients are transported from the dermis by diffusion. Therefore, epidermal cell metabolism should be considered a unique and sensitive marker of early dysfunction in vascular circulation and metabolic regulation.

The efforts of the authors of the present contribution have resulted in a new diagnostic technique named Flow Mediated Skin Fluorescence (FMSF), which enables non-invasive evaluation of the vascular circulation and metabolic regulation. ${ }^{1,2}$ The FMSF technique is based on monitoring the intensity of NADH fluorescence from skin tissue on the forearm in response to blocking and releasing blood flow, as a function of time. Measurements are performed using the AngioExpert, a diagnostic device constructed by Angionica Ltd. The FMSF technique has been tested on various pathologies and disorders, as well as in sport physiology. ${ }^{3-6}$

Oscillations in the microcirculation, known as flowmotion, are a wellrecognized characteristic of cutaneous blood flow. ${ }^{7}$ Very recently, we demonstrated that skin flowmotion can be monitored very distinctly and precisely using the FMSF technique. ${ }^{8}$ The FMSF technique appears to be uniquely suited to the analysis of basal flowmotion and its hypoxia response, and may be used for characterization of microcirculatory status.

Two different periods of oscillations can be distinguished in the FMSF signal: basal oscillations at rest, and flowmotion during the reperfusion stage. Analysis of flowmotion on the reperfusion line following post-occlusive reactive hyperemia (PORH) allows for quantitative assessment of the patient's reaction to hypoxia caused by transient ischemia. Flowmotion parameters characterizing the microcirculatory response to hypoxia have been found to be impaired among patients with various diseases and disorders, including hypertension, diabetes, and cardiovascular disease. Flowmotion parameters have also been found to be dependent on age and blood pressure.

Several quantitative measures of oscillations have been defined:

- FM - a parameter characterizing basal flowmotion at rest;

- $\mathrm{FM}(\mathrm{R})$ - a parameter representing flowmotion during the reperfusion phase, which reflects the strong effect of hypoxia on flowmotion, mainly due to the increased activity of the vessels; 
- HS (Hypoxia Sensitivity) - a fraction of FM(R) value covering the intensity of flowmotion related to myogenic oscillations $(0.052-0.15 \mathrm{~Hz})$ recorded during reperfusion. Since myogenic oscillations are predominantly stimulated on the reperfusion line following transient ischemia, the HS parameter seems to be particularly well-suited for quantitative characterization of the microcirculatory response to hypoxia.

Full details of the flowmotion analysis and its diagnostic application are available elsewhere. ${ }^{8}$

It is postulated that the hypoxia-inducible factor (HIF) plays a prominent role in the body's response to hypoxia. Stabilization of HIF-1 $\alpha$ induces a number of signaling pathways responsible for the regulation of many physiologic processes, such as the innate immune response, wound healing and adaptation to high-altitudes or physical exercise. It is assumed that such responses may be regulated by genetic factors. High values of the measured HS parameter seem to reflect an efficient stabilization of HIF-1 $\alpha$ in microvascular smooth muscle cells during transient hypoxia.

In this paper, we present an analysis of the HS parameter measured for three studied groups: highly trained athletes (group A), healthy controls (group B) and diabetes type 2 patients (group C). The study was conducted at the Medical University of Lodz, Poland, and Poznan University of Physical Education, Poland. It conformed to the principles outlined in the Declaration of Helsinki and the study protocol was approved by the University Bioethics Committee. All the subjects gave written informed consent prior to participation. The individuals participating in the study were tested twice using the AngioExpert device. Average values of the measured parameters were used in the analysis.

Exemplary FMSF traces recorded for two individuals are presented in Figure 1. It is clearly seen that the highly trained athlete has very high sensitivity to hypoxia measured by the HS parameter and also $\mathrm{FM}(\mathrm{R})$ parameter is found to be much higher compared to FM parameter (Figure 1A). In contrast, the diabetes type 2 patient has very low sensitivity to hypoxia and all three FM, FM(R) and HS parameters are very low (Figure 1B).

Based on FMSF studies involving several hundred patients, we decided to assign the participating individuals to four ranges of HS values, describing distinguishable levels of microvascular function (very low, low, moderate and high), as shown in Figure 2.

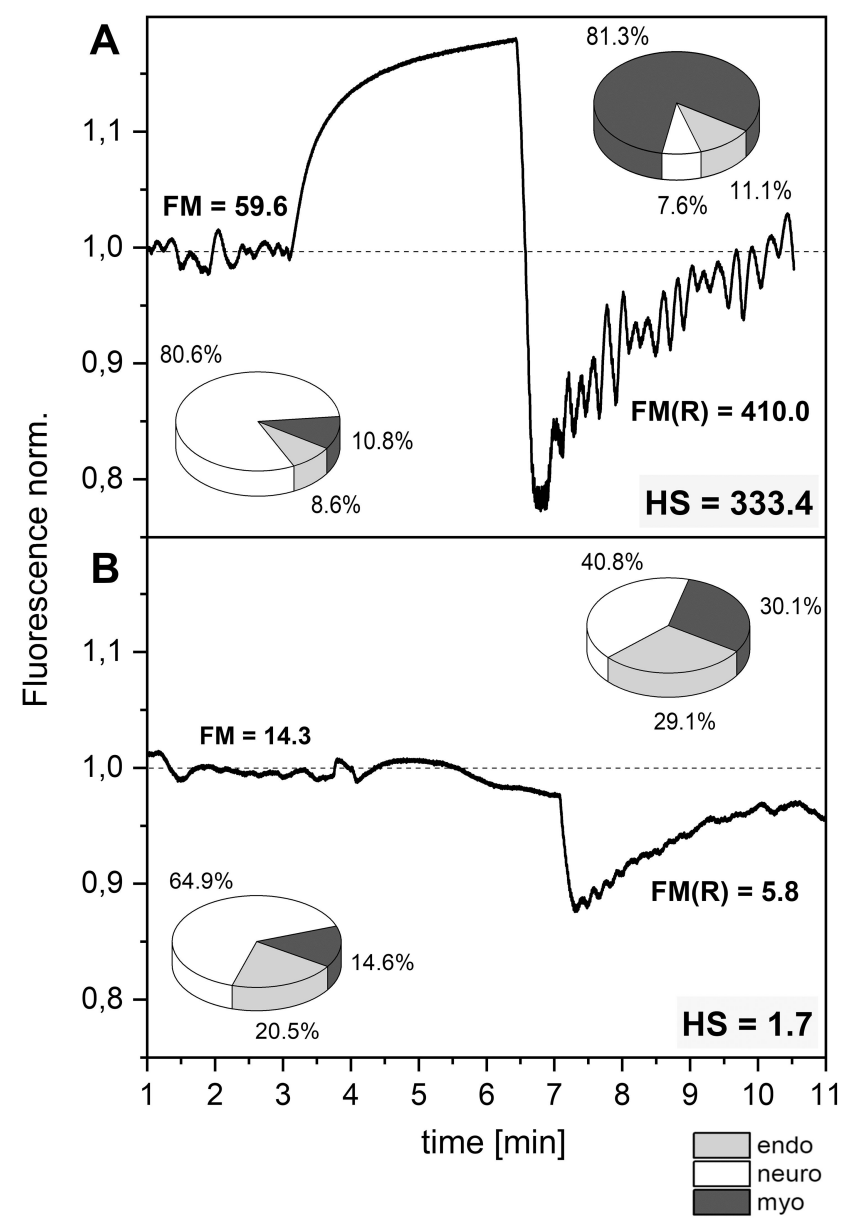

Figure I Exemplary FMSF traces recorded for two individuals: (A) highly trained athlete with very high sensitivity to hypoxia (male, age 24 y., long-distance runner), (B) diabetes type 2 patient with very low sensitivity to hypoxia (male, age $70 \mathrm{y}$., disease duration 20 y., retinopathy, neuropathy). Relative contribution to the lowfrequency oscillations: endo-endothelial, neuro-neurogenic and myo-myogenic.

Important conclusions can be drawn from the results presented in Figure 2. In group A, which was composed of highly trained athletes (long-distance runners -9 , sprinters -7 , rowers -9 and taekwondo practitioners -8 ), $63.6 \%$ of individuals had a high level of microvascular circulation. Indeed, $30.3 \%$ of the whole group had HS values above 200 , and their microvascular circulation can be described as excellent. Due to their efficient vascular systems, these individuals have strong potential to be outstanding sportsmen. Only in this group were individuals identified with HS $>200$. We also identified two individuals in group A with weak microvascular function, one of whom who had a low HS level (HS $=17.42$, male, 19 y., taekwondo) and the other a very low HS level ( $\mathrm{HS}=8.58$, male, 18 y., sprinter). Clearly, the health status of these individuals should be examined carefully before continuation of a sports career. 


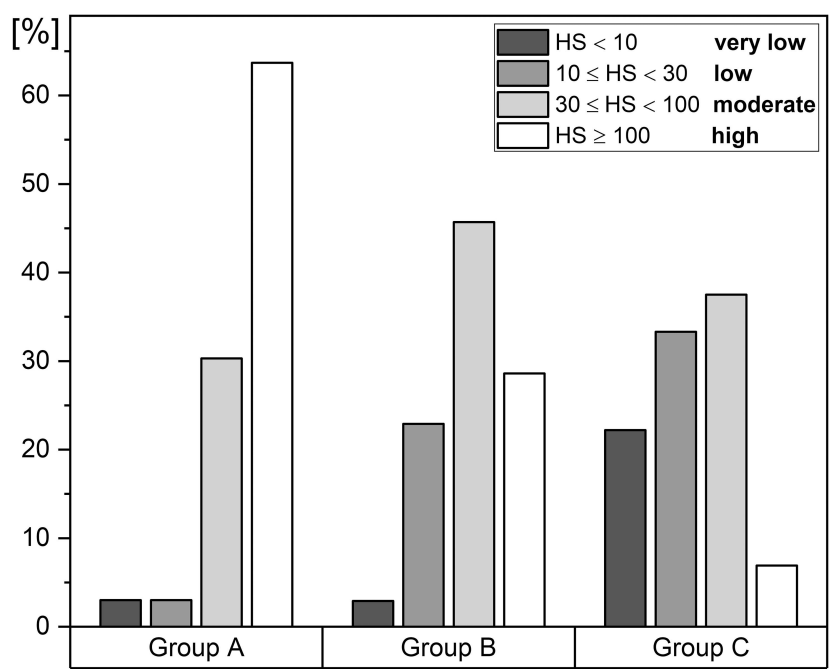

Figure 2 Distribution of the HS parameter in groups (A-C) (A) highly trained athletes, $n=33(26 \mathrm{~m}, 7 \mathrm{f})$, mean age 22.5 (16-35 y.), (B) healthy middle-aged individuals, $n=35(22 \mathrm{~m}, 13 \mathrm{f})$, mean age 38.5 (30-50 y.), (C) diabetes type 2 patients, $n=72(40 \mathrm{~m}, 32 \mathrm{f})$, mean age $62(50-80 \mathrm{y}$ ) $)$. The statistical significance of differences between parameters for the compared groups (Kruskal-Wallis test): $A$ vs $B, p<0.01 ; A$ vs C, $p<I E(-9) ; B$ vs $C, p<0.005$.

A strikingly different picture emerges from analysis of group C (diabetes type 2 patients). In this group, 55.5\% had very low or low values for the HS parameter. We are convinced that it is possible to predict microvascular complications associated with diabetes based on the HS parameter and such studies have already been initiated. Only 5 out of 72 patients in group $\mathrm{C}$ had high values for HS $(>100)$. Microvascular complications are unlikely to develop in these patients, despite long disease duration and advanced age. This phenomenon seems to point towards a genetic predisposition.

Analysis of group B (healthy middle-aged individuals, $30-50$ y.) reveals that 8 individuals ( 2 female and 6 male) had low values for the HS parameter, and one individual (male) had a very low HS parameter. It is not surprising that the individuals from groups $\mathrm{A}$ and $\mathrm{B}$ with low or very low HS parameters were predominantly males ( 9 out of 11). The existence of gender differences in vascular function is well known, and it is thought that sex hormones play a crucial role.

The analysis presented above suggests that the HS parameter is uniquely suited for assessment of microvascular function. The broad window for analysis of the HS parameter makes this approach applicable to both healthy individuals and patients with evident impairment of microvascular function.

Very recently, we proposed the use of the microcirculatory response to hypoxia measured by the FMSF technique as a prognostic factor for Covid-19. ${ }^{9}$ We believe that measuring the HS parameter could be useful for predicting the severity of Covid-19 symptoms, particularly considering the recent observation that hypoxemia is independently associated with Covid-19 in-hospital mortality.

\section{Funding}

This work was supported by the European Union from the resources of the European Regional Development Fund under the Smart Growth Operational Program, Grant No. POIR. 01.01.01-00-0540/15-00.

\section{Disclosure}

JG and AM are inventors of the patents protecting the use of FMSF technology. JG and AM have a patent issued to PL, EU, USA, JPN, CAN, AUS, China, and Russia. The authors report no other conflicts of interest in this work.

\section{References}

1. Katarzynska J, Lipinski Z, Cholewinski T, et al. Non-invasive evaluation of microcirculation and metabolic regulation using flow mediated skin fluorescence (FMSF): technical aspects and methodology. Rev Sci Instrum. 2019;90:104104. doi:10.1063/1.5092218

2. Hellmann M, Tarnawska M, Dudziak M, et al. Reproducibility of flow mediated skin fluorescence to assess microvascular function. Microvasc Res. 2017;113:60-64. doi:10.1016/j.mvr.2017.05. 004

3. Katarzynska J, Borkowska A, Czajkowski P, et al. Flow Mediated Skin Fluorescence technique reveals remarkable effect of age on microcirculation and metabolic regulation in type 1 diabetes. Microvasc Res. 2019;124:19-24. doi:10.1016/j.mvr.2019.02.005

4. Katarzynska J, Borkowska A, Los A, et al. Flow-Mediated Skin Fluorescence (FMSF) technique for studying vascular complications in type 2 diabetes. J Diab Sci Technol. 2020;14:693-694. doi:10.1177/ 1932296819895544

5. Majewski S, Szewczyk K, Bialas AJ, et al. Assessment of microvascular function in vivo using flow mediated skin fluorescence (FMSF) in patients with obstructive lung diseases: A preliminary study. Microvasc Res. 2020;127:103914. doi:10.1016/j.mvr.2019.103914

6. Bugaj O, Zielinski J, Kusy K, et al. The effect of exercise on the skin content of the reduced form of NAD and its response to transient ischemia and reperfusion in highly trained athletes. Front Physiol. 2020;10:600. doi:10.3389/fphys.2019.00600

7. Rossi M, Capri A, Galetta F, et al. The investigation of skin blood flowmotion: a new approach to study the microcirculatory impairment in vascular diseases? Biomed Pharmacother. 2006;60:437-442. doi:10.1016/j.biopha.2006.07.012

8. Gebicki J, Katarzynska J, Cholewinski T, et al. Flowmotion monitored by Flow Mediated Skin Fluorescence (FMSF): a tool for characterization of microcirculatory status. Front Physiol. 2020;11:702. doi:10.3389/fphys.2020.00702

9. Gebicki J, Katarzynska J, Marcinek A. Can the microcirculatory response to hypoxia be a prognostic factor for Covid-19? Respir Physiol Neurobiol. 2020;280:103478. doi:10.1016/j.resp.2020.103 478 


\section{Publish your work in this journal}

Vascular Health and Risk Management is an international, peerreviewed journal of therapeutics and risk management, focusing on concise rapid reporting of clinical studies on the processes involved in the maintenance of vascular health; the monitoring, prevention and treatment of vascular disease and its sequelae; and the involvement of metabolic disorders, particularly diabetes. This journal is indexed on PubMed Central and MedLine. The manuscript management system is completely online and includes a very quick and fair peerreview system, which is all easy to use. Visit http://www.dovepress. com/testimonials.php to read real quotes from published authors. 